Supporting Information

\title{
All-Elastomer-Based Triboelectric Nanogenerator as a Keyboard Cover to Harvest Typing Energy
}

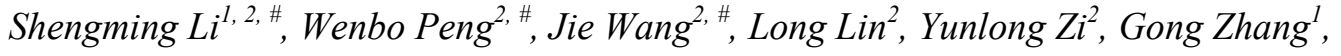 \\ Zhong Lin Wang ${ }^{2,3, *}$ \\ ${ }^{1}$ Department of Mechanical Engineering, Tsinghua University, Beijing 100084, China \\ ${ }^{2}$ School of Materials Science and Engineering, Georgia Institute of Technology, Atlanta, Georgia 30332- \\ 0245, United States \\ ${ }^{3}$ Beijing Institute of Nanoenergy and Nanosystems, Chinese Academy of Sciences, Beijing 100083, \\ China \\ \# These authors contributed equally. \\ * Corresponding author: e-mail: zhong.wang@mse.gatech.edu
}

\section{Contents}

A: The finite element modelling results of the electric output for single-electrode mode TENGs with different gap distances and constant upper layer thickness by COMSOL

B: The finite element modelling results of the electric output for contact-separation mode TENGs with different gap distances and constant upper layer thickness by COMSOL.

C: The finite element modelling results of the electric output for single-electrode mode TENGs with different upper layer thicknesses and constant gap distance by COMSOL.

D: Output power and current under different loading resistance.

E: The circuit diagram of the self-charging power system integrated with the TENG and the supercapacitor.

F: Capacitance properties of the h-PPy based supercapacitor.

G: The $V_{\text {Charging }}-t$ curve of the supercapacitor while powering an electronic thermometer/hygrometer.

H: Comparison of electric outputs by typing with/without nitrile gloves on. 


\section{A: The finite element modelling results of the electric output for single-electrode mode}

TENGs with different gap distances and constant upper layer thickness by COMSOL.

The simulation results by COMSOL are presented here with the Normalized $\Delta Q_{S C}-d$ relationship when $D$ is $0.8 \mathrm{~mm}$. We also presented the the potential distribution for states when: (1) The hand is far away from the TENG; (2) The hand firmly touches the surface of the TENG.

a

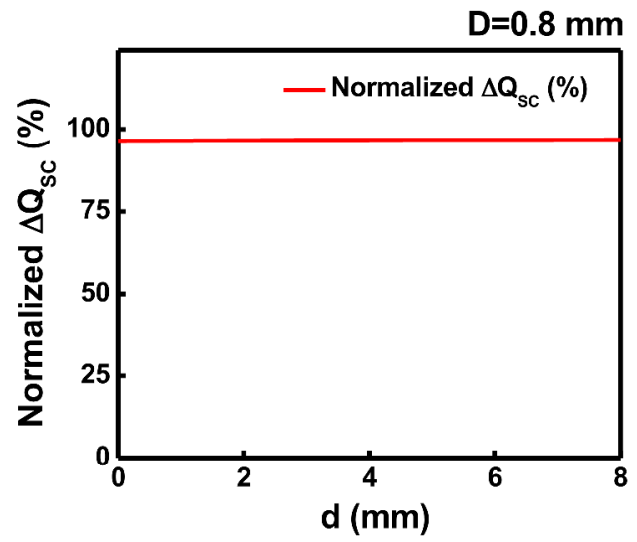

b

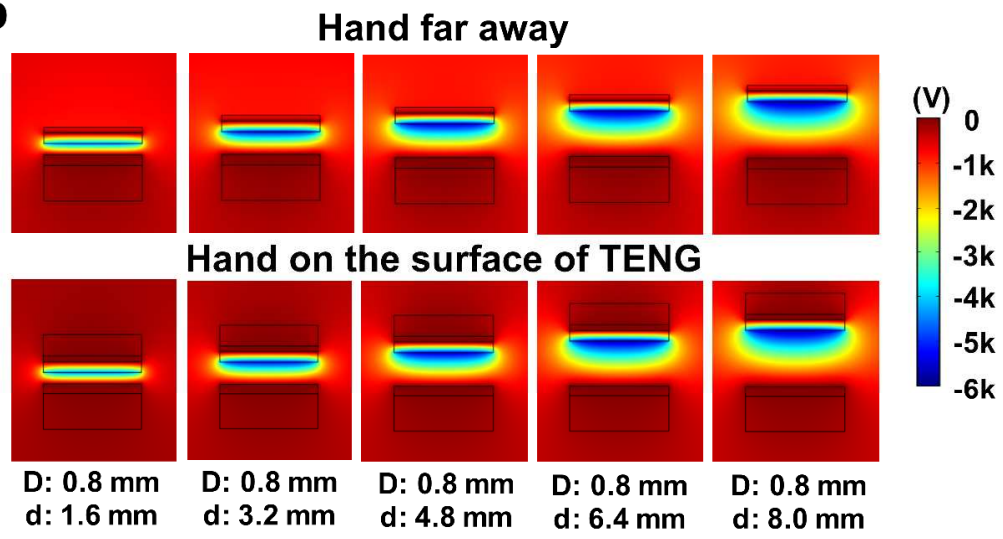

Figure S1. The finite element modelling results of the electric output for single-electrode mode TENGs with different gap distances by COMSOL. (a) The Normalized $\Delta Q_{S C}-d$ relationship with $D=0.8 \mathrm{~mm}$ and the gap distance constant while the hand is approaching and leaving from the TENG by COMSOL. (b) The potential distributions of the states when: (1) the hand is far away from the TENG; (2) the hand firmly touches the surface of the TENG with $\mathrm{D}=0.8 \mathrm{~mm}$ and the different constant gap distances. 


\section{B: The finite element modelling results of the electric output for contact-separation mode}

TENGs with different gap distances and constant upper layer thickness by COMSO.

The simulation results by COMSOL are presented here with the Normalized $\Delta Q_{S C}-d$ relationship when $D$ is $0.8 \mathrm{~mm}$. We also presented the the potential distribution for states when: (1) The air gap distance is 0 ; (2) The hand touches the surface of the TENG with no elastic deformation (maximum gap distance).

a

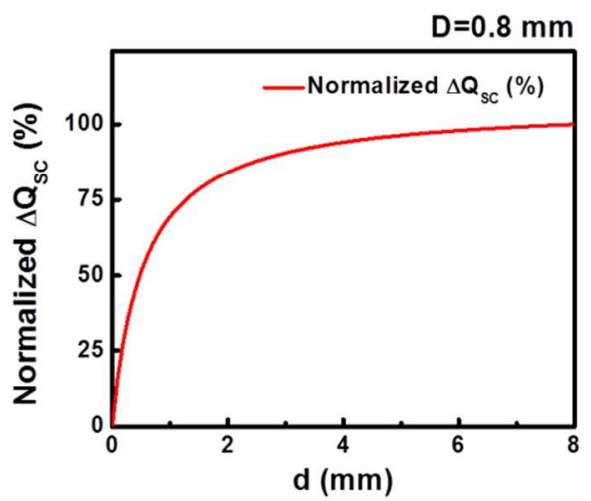

b

Full contact
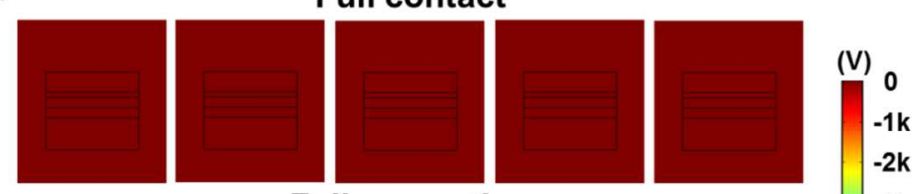

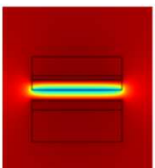

D: $0.8 \mathrm{~mm}$ d: $1.6 \mathrm{~mm}$

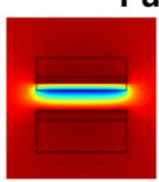

D: $0.8 \mathrm{~mm}$ d: $3.2 \mathrm{~mm}$
Full separation

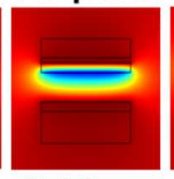

D: $0.8 \mathrm{~mm}$ d: $4.8 \mathrm{~mm}$

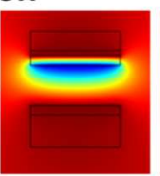

D: $0.8 \mathrm{~mm}$ d: $6.4 \mathrm{~mm}$

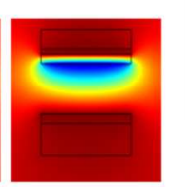

D: $0.8 \mathrm{~mm}$ d: $8.0 \mathrm{~mm}$

Figure S2. The finite element modelling results of the electric output for contact-separation mode TENGs with different gap distances by COMSOL. (a) The Normalized $\Delta Q_{S C}-d$ relationship with $D=0.8 \mathrm{~mm}$ and the hand contact closely with the surface of TENG while the gap distance is changing by COMSOL. (b) The potential distributions of the states when: (1) the air gap distances are 0 ; (2) the air gap distances are maximum for TENGs with $D=0.8 \mathrm{~mm}$ and the different maximum air gap distances. 
C: The finite element modelling results of the electric output for single-electrode mode TENGs with constant gap distance and different upper layer thicknesses by COMSOL.

The simulation results by COMSOL are presented here with the Normalized $\Delta Q_{S C}-D$ relationship when $d$ is $0.8 \mathrm{~mm}$. We also presented the the potential distribution for states when: (1) The hand is far away from the TENG; (2) The hand firmly touches the surface of the TENG.

a

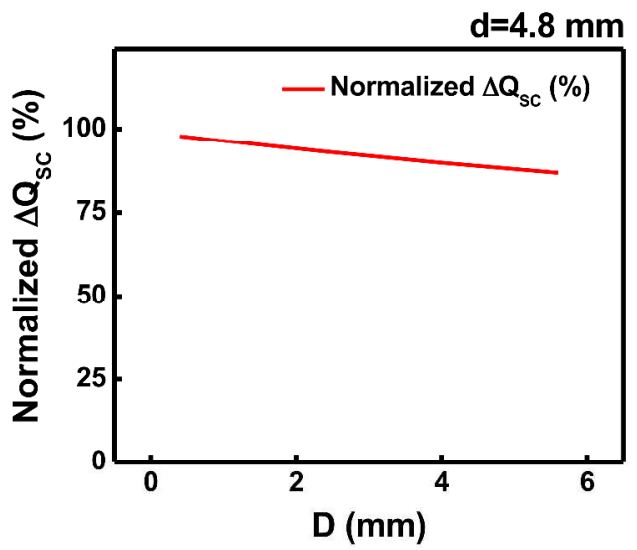

b
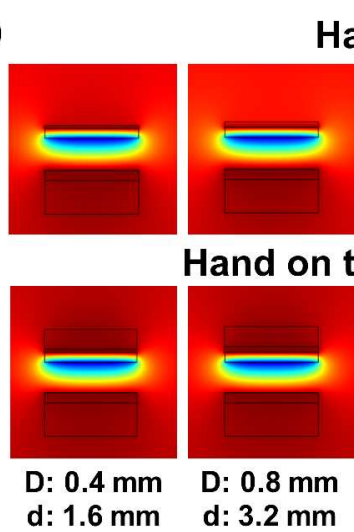

D: $0.8 \mathrm{~mm}$ d: $3.2 \mathrm{~mm}$

Hand far away
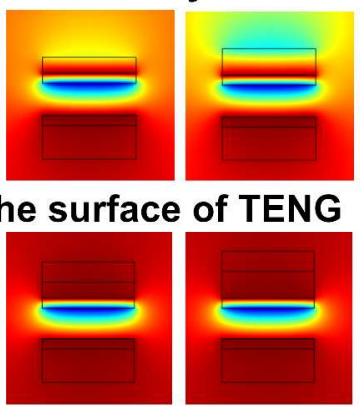

D: $2.4 \mathrm{~mm}$ d: $4.8 \mathrm{~mm}$

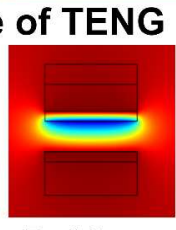

D: $4.0 \mathrm{~mm}$ d: $6.4 \mathrm{~mm}$

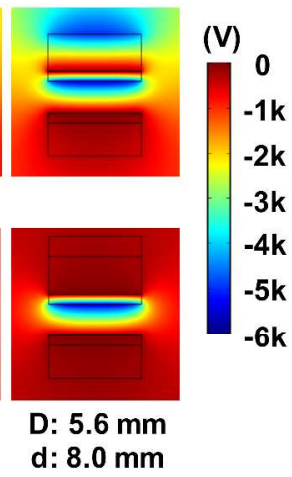

Figure S3. The finite element modelling results of the electric output for single-electrode mode TENGs with different upper structural layer thicknesses by COMSOL. (a) The Normalized $\Delta Q_{S C}-D$ relationship with $d=4.8 \mathrm{~mm}$ while the hand is approaching and leaving from the TENG by COMSOL. (b) The potential distributions of the states when: (1) the hand is far away from the TENG; (2) the hand firmly touches the surface of the TENG with $d=4.8 \mathrm{~mm}$ and the different upper layer thicknesses. 
D: Output power and current under different loading resistance.

Here we demonstrate the output power density and current density versus different loading resistance under the frequency of $3 \mathrm{~Hz}$.

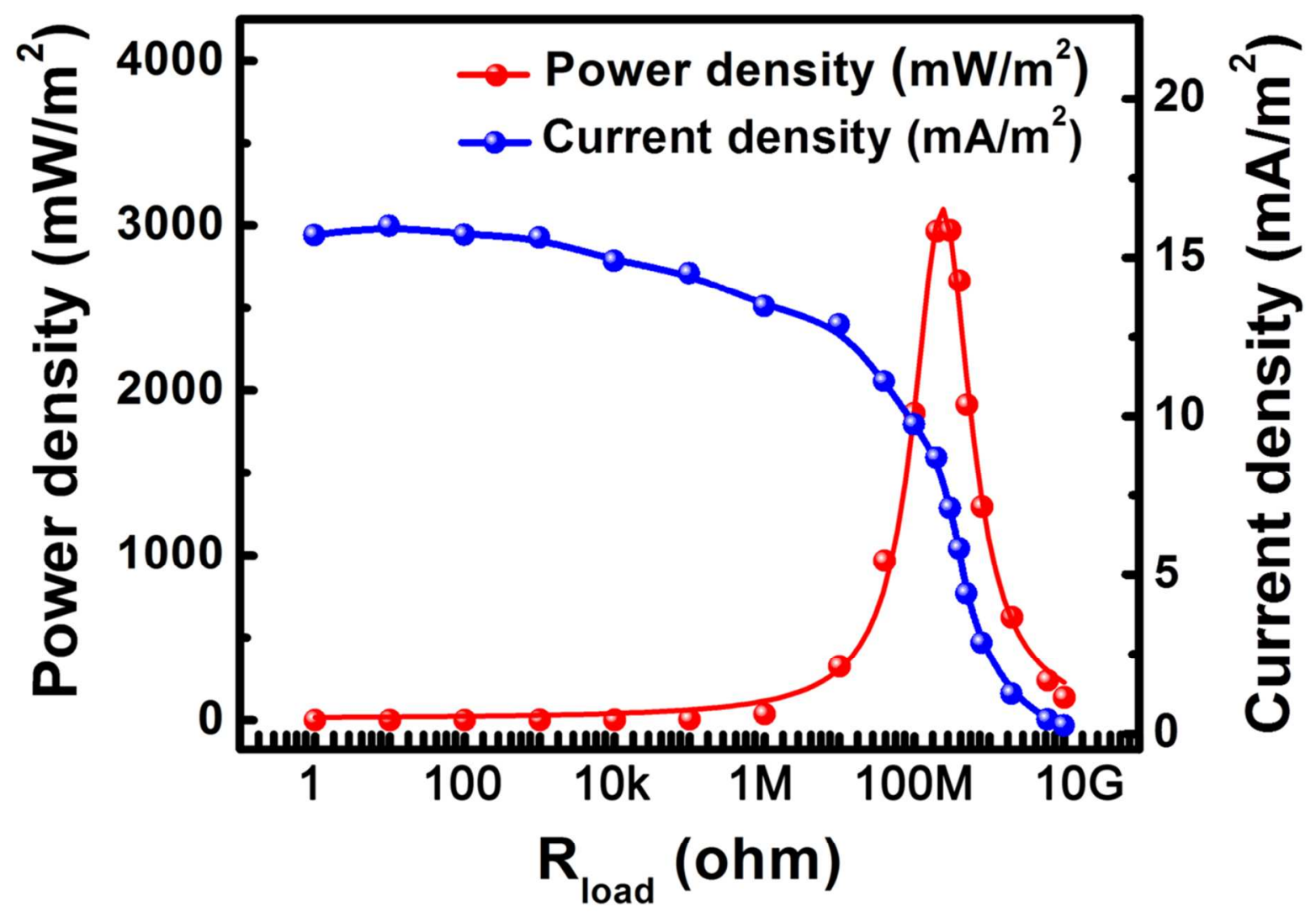

Figure S4. Output power density and current density versus loading resistance. 
E: The circuit diagram of the self-charging power system integrated with the TENG and the supercapacitor.

The circuit diagram of the self-charging power system is demonstrated in Figure S4.

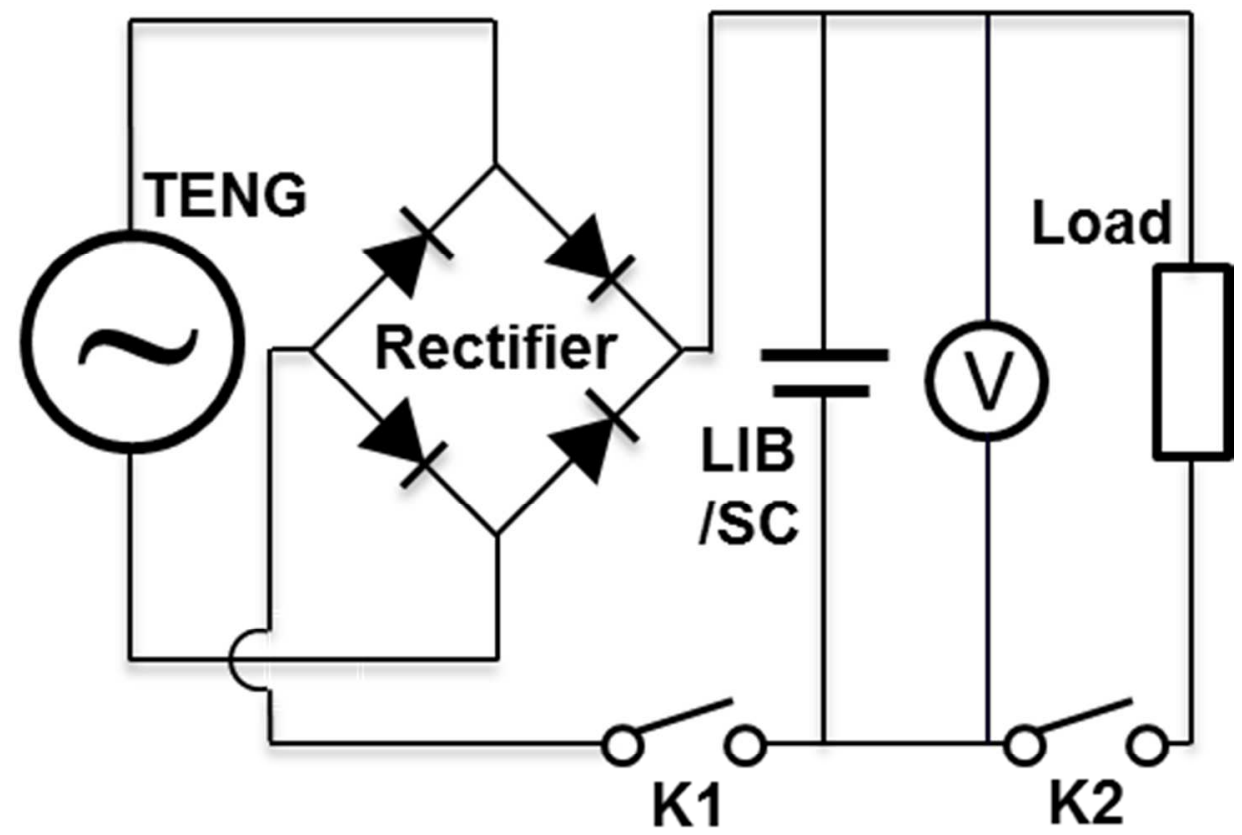

Figure S5. The circuit diagram of the self-charging power system integrated with the TENG and the supercapacitor. 


\section{F: Capacitance properties of the h-PPy based supercapacitor.}

The capacitance properties of the h-PPy based supercapacitor are demonstrated by the electrochemical impedance spectroscopy with an inset plot of capacitance versus frequency and the GCD curves at the current loads of $2 \mu \mathrm{A}$ and $10 \mu \mathrm{A}$.
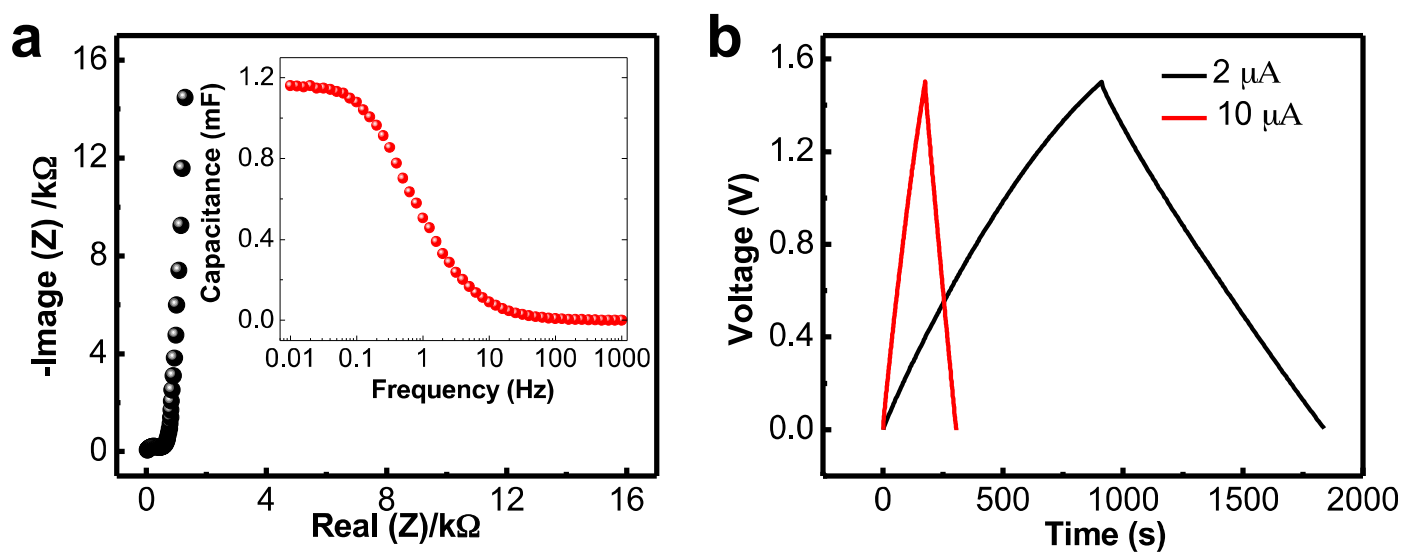

Figure S6. Capacitance properties of the h-PPy based supercapacitor (a) Electrochemical impedance spectroscopy, with an inset plot of capacitance versus frequency. (b) The GCD curves at the current loads of $2 \mu \mathrm{A}$ and $10 \mu \mathrm{A}$. 
G: The $V_{\text {Charging }}-t$ curve of the supercapacitor while powering an electronic thermometer/hygrometer.

Here we demonstrate the voltage of the supercapacitor while powering the thermometer/hygrometer. The electronic thermometer/hygrometer could be driven when the supercapacitor is charged to $\sim 1.15 \mathrm{~V}$. Charging the super-capacitor to $\sim 1.6 \mathrm{~V}$, the thermometer/hygrometer could be powered for 2 minutes. Figure S8 demonstrated the discharging process of the super-capacitor while powering the thermometer/hygrometer.

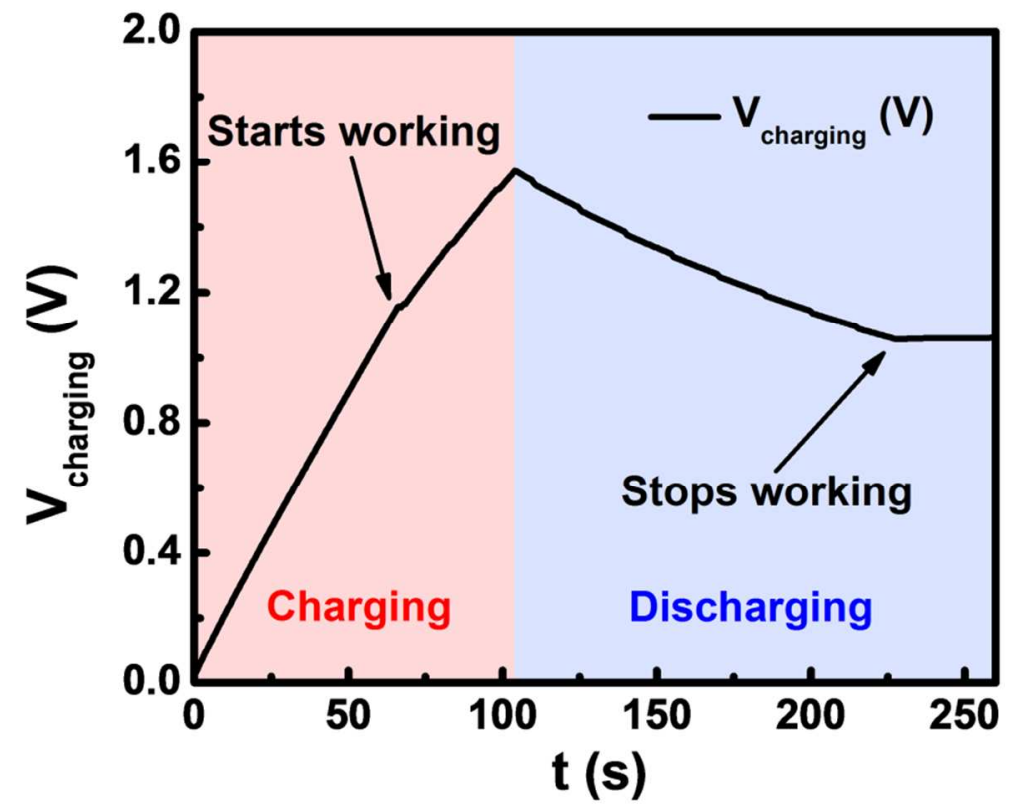

Figure S7. The $V_{\text {Charging }}-t$ curve of the supercapacitor while powering an electronic thermometer/hygrometer. 
H: Comparison of electric outputs by typing with/without nitrile gloves on.

Here we compare the electric outputs by typing with/without nitrile gloves on by presenting the $\Delta Q_{S C}-t$ curves for both conditions.

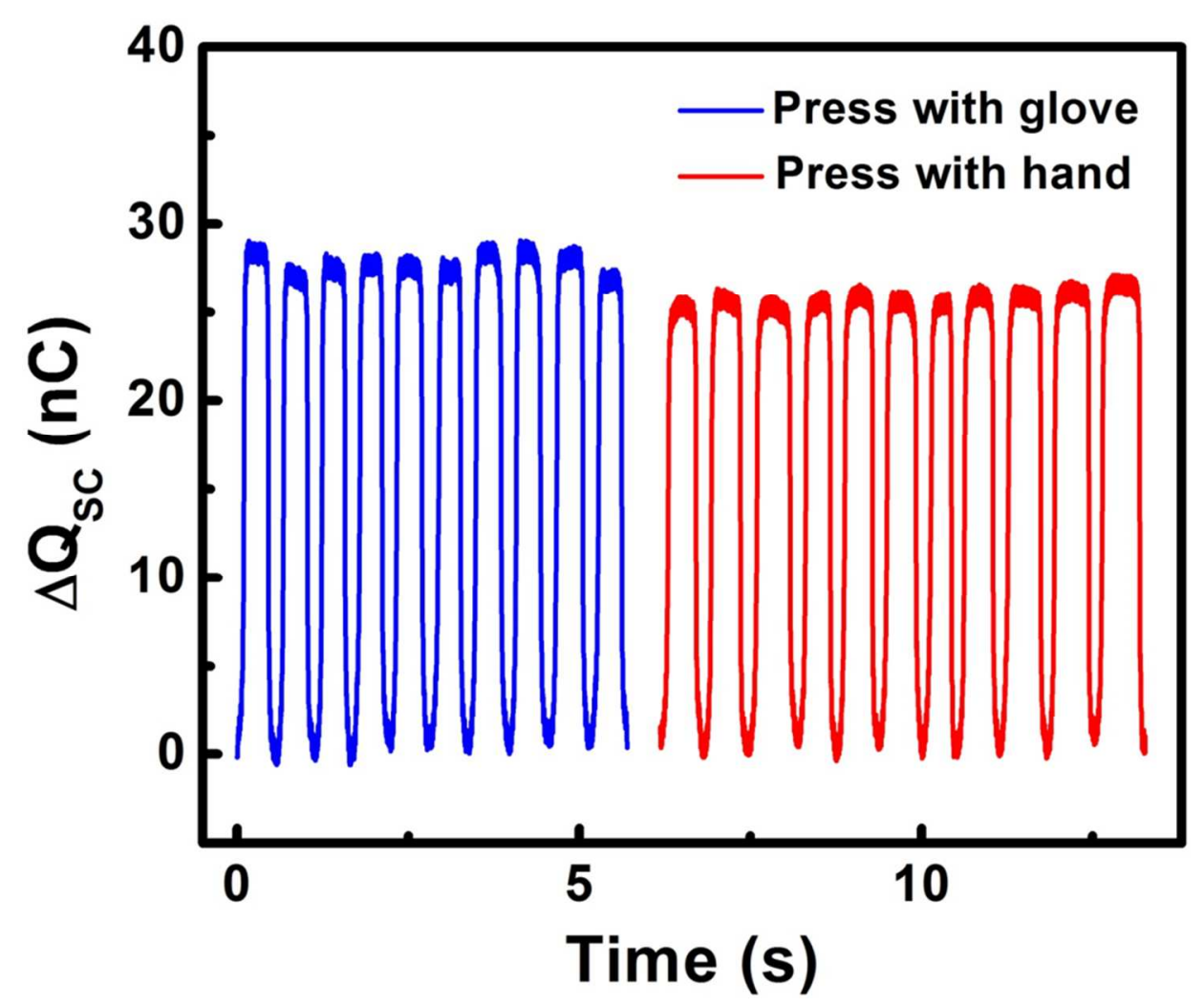

Figure S8. $\Delta Q_{S C}-t$ curves by typing letter " $\mathrm{T}$ " fully with/without nitrile gloves on. 